\title{
Multivariate Analysis Applications in ToF-SIMS
}

\author{
Robert M. Moision" ${ }^{1}$, John A. Chaney ${ }^{1}$ \\ ${ }^{1}$ The Aerospace Corporation, Electronics and Photonics Laboratories, El Segundo USA.
}

As the size and complexity of data sets generated by modern instrumentation continue to grow, more emphasis is being placed on how to efficiently ascertain and characterize underlying processes and relationships responsible for observed behavior. Multivariate analysis (MVA) techniques are increasingly relied upon to provide a means of analyzing and understanding large and often multidimensional data sets. MVA is a general term and includes a number of statistical techniques (and bewildering acronyms) that can be used for identification, classification, and prediction. In this work, three ToF-SIMS examples will be used to showcase how different MVA approaches have been applied to root cause investigations using surface and subsurface ToF-SIMS data sets. The emphasis will be on how to extract and organize data to maximize the application of MVA techniques and how MVA can provide insight to problems that would be difficult to approach using traditional techniques. While MVA will be discussed in the context of ToF-SIMS, the methods discussed will be applicable to a number of analytical approaches.

Example 1. During the startup test and commissioning, the primary mirror on the visible/infared imager radiometer suite (VIIRS) onboard the Suomi national polar-orbiting partnership (Suomi-NPP) satellite exhibited darkening in the infared bands. ToF-SIMS depth profiles were used to assess the chemical and physical similarity of two witness mirrors, one pristine (FabWit) and one attached to the satellite during construction (TWM). The TWM mirror was exposed to a number of environmental contaminants during satellite assembly making a straightforward comparison to the pristine FabWit mirror difficult. Multivariate curve resolution (MCR) was employed to factor out the contamination layer on the TWM, allowing for straightforward comparison of the remaining mirror layers. The results conclusively showed that the TWM and and FabWit mirrors were similar, providing a key piece of evidence during the root cause investigation.

Example 2. Particle counts on a hardware surface of a launch vehicle exceeded threshold requirements in six successive tests despite repeated cleaning. ToF-SIMS analysis was performed on tape-lift samples to provide chemical identity of the particles. The tape-lifted particles were not optically visible and produced a very low intensity ToF-SIMS signals, making them difficult to identify during experimentation. Prior to MVA analysis, high resolution mass spectral data was condensed by over a factor of 200 into 1 amu sized bins and the original $256 \times 256$ pixel image was also resampled to a $64 \times$ 64 pixel image. Despite the loss of resolution in mass and spatial dimensions, MVA analysis detected spatially correlated signals consistent with expected particle size. Using MVA results along with the raw data revealed the particles to be alkylbenzene sulfonates, a common surfactant used in detergents.

Example 3. Titanium based hydrogen getters were subjected to repeated $\mathrm{H}_{2}$ exposure and baking cycles to test their uptake efficiency. Following baking, the getters were reported to have been "rejuvenated", despite the bake temperature being well below that necessary to remove hydrogen from bulk titanium. ToF-SIMS depth profiles of the getters were used to detect any chemical changes to the structure that might explain the improved getter response following baking. Partial least squares (PLS) analysis was used to search for mass spectral peaks that might be correlated to the hydrogen exposure levels. A peak 
at 32 amu was found to be positively correlated with hydrogen exposure and baking cycles. A more detailed analysis of the raw data revealed that sulfur, not molecular oxygen was responsible for the signal change at 32 amu.

a)

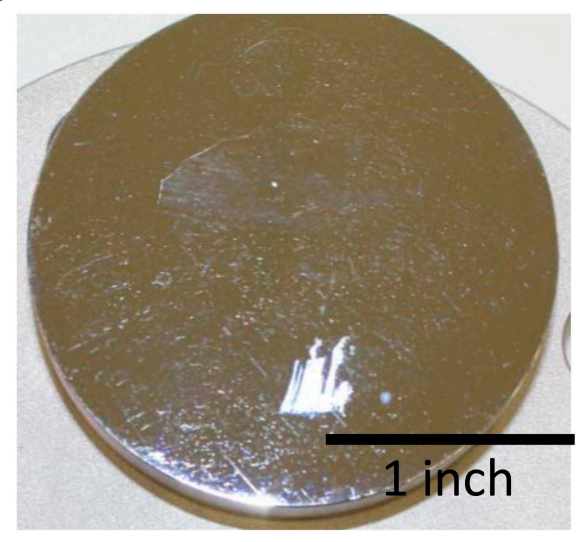

b)

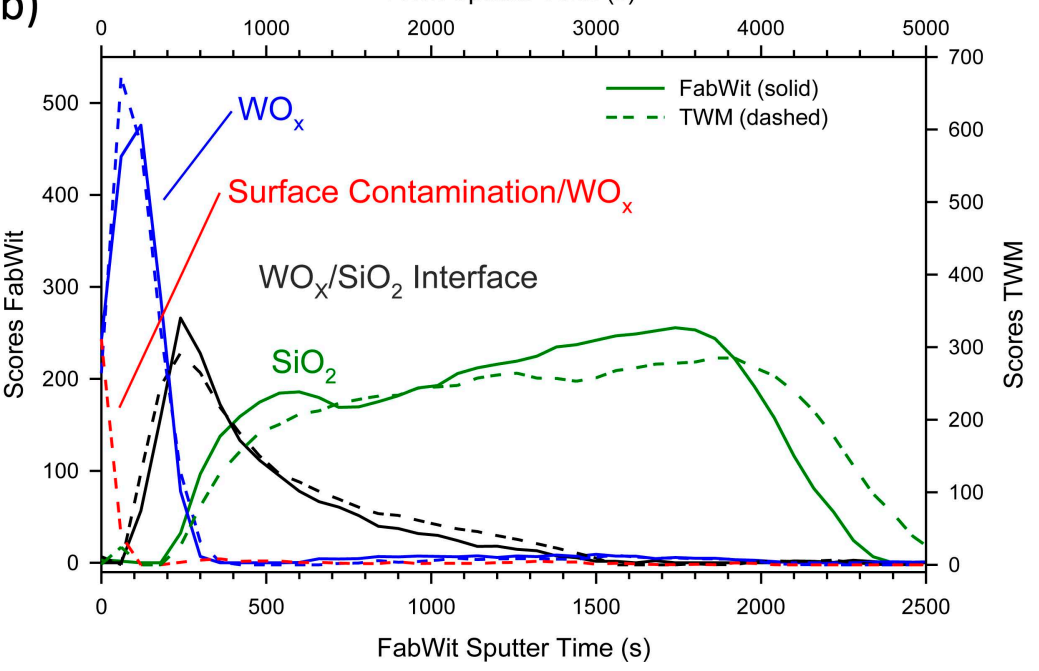

Figure 1. a) Telescope witness mirror (TWM) from VIIRS with visible scratches and staining due to exposure and handling. b) Comparison of multivariate curve resolution components of TWM and pristine FabWit mirrors display a high degree of similarity. Data for TWM has been modeled with one additional component to describe surface contamination. Mass spectral signatures associated with each component also show high similarity.

a)

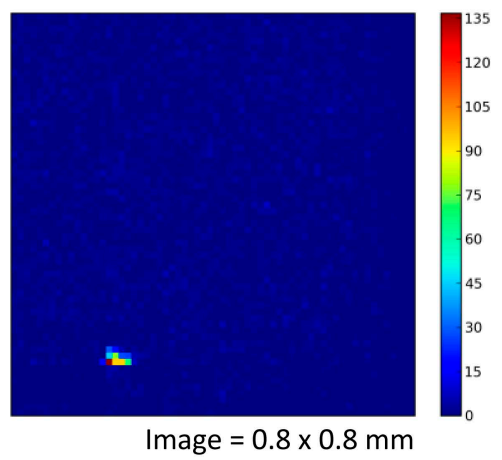

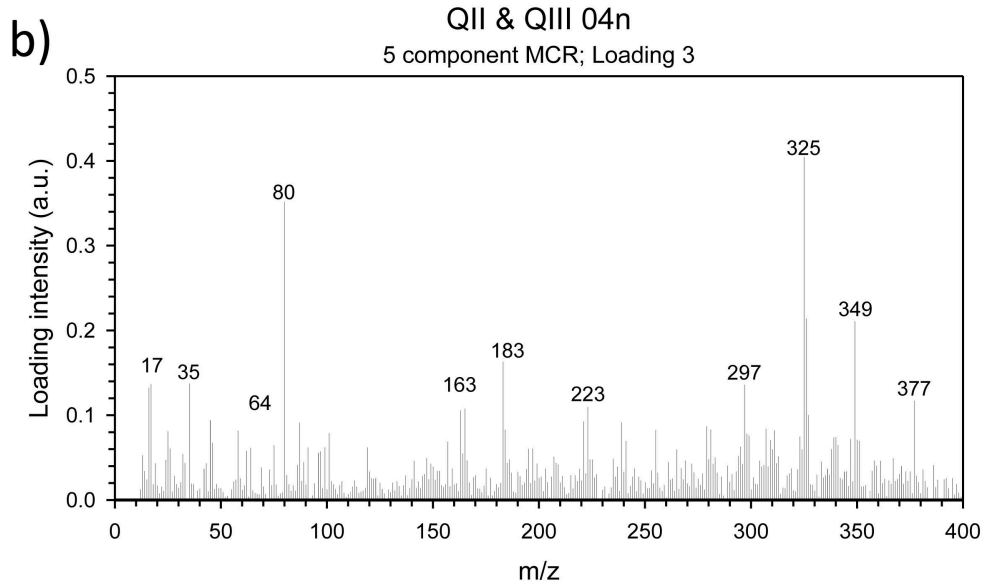

Figure 2. a) Image showing multivariate component of particle from tape lift. b) Spectral signature of the particle component has peaks consistent with alkybenzene sulfonate. 